\title{
Alpha-2 Receptor Blockade Increases Responsiveness of Locus Coeruleus Neurons to Excitatory Stimulation
}

\author{
Peter E. Simson and Jay M. Weiss \\ Department of Psychiatry, Duke University Medical Center, Durham, North Carolina 27710
}

This study presents evidence that $\alpha_{2}$-receptors in the locus coeruleus (LC) regulate the responsiveness of LC neurons to excitatory stimuli. In the first experiment, intravenous administration of the $\alpha_{2}$-adrenergic antagonist idazoxan markedly potentiated the responses of LC neurons to the excitatory stimulus of contralateral hind paw compression (PC). Increased responsiveness of LC neurons to PC was seen with doses of idazoxan far below those that altered spontaneous activity of the LC. In the second experiment, increased responsiveness of LC neurons to PC was seen when low doses of idazoxan were infused directly into the LC, thereby indicating that increased responsiveness of LC neurons resulted from blockade of $\alpha_{2}$-receptors in the LC region and not from greater stimulus input to the $L C$ resulting from blockade of $\alpha_{2}$-receptors elsewhere. In the third experiment, another $\alpha_{2}$-adrenergic antagonist, yohimbine, also increased the responsiveness of LC neurons to PC. Finally, the response of the $L C$ to another excitatory stimulus, peripheral injection of nicotine, was also found to be increased by idazoxan. Results obtained prior to these studies had indicated that $\alpha_{2}$-receptors in the LC regulate the period of quiescence that follows a burst of LC firing. However, more recent studies suggest that this quiescence results primarily from changes in ionic conductance of the membrane following directly from depolarization. The present findings indicate that, in addition to whatever role $\alpha_{2}$-receptors play in regulating postfiring quiescence, these receptors in the LC appear to play a major role in regulating the responsiveness of LC neurons to excitational influences.

The locus coeruleus (LC) is a tightly packed pontine nucleus containing approximately half of all noradrenergic neurons in the rat brain (Nygren and Olson, 1977). Pioneering pharmacological studies by Aghajanian and his colleagues established that $\alpha_{2}$-receptors, which have since been found in high density in the LC (Young and Kuhar, 1980), inhibit the firing of LC neurons. These investigators showed that stimulation of $\alpha_{2}$-receptors by systemic or iontophoretic administration of the $\alpha_{2}-$ adrenergic agonist clonidine inhibited LC neuronal activity in

\footnotetext{
Received June 30, 1986; revised Dec. 17, 1986; accepted Dec. 17, 1986.

This research was supported by Public Health Service Grant MH 40406 and a Neurobehavioral Science Research Training Program Fellowship (MH-15177). We gratefully acknowledge Dr. T. Celeste Napier for her consultation and guidance throughout this project. We wish to thank Jeffrey A. Knight for his technical assistance. We also thank Reckitt and Colman, Kingston-upon-Hull, England, for their generous gift of idazoxarı hydrochloride.

Correspondence should be addressed to Peter E. Simson, Ph.D., Department of Psychiatry, Duke University Medical Center, Box 3829, Durham, NC 27710.

Copyright $(\overline{\mathcal{C}}) 1987$ Society for Neuroscience $0270-6474 / 87 / 061732-09 \$ 02.00 / 0$
}

a dose-dependent manner (Svensson et al., 1975). In addition, it was shown that the inhibition of the LC by iontophoretic application of NE (Svensson et al., 1975; Cedarbaum and Aghajanian, 1976) and epinephrine (E) (Cedarbaum and Aghajanian, 1976) was blocked by pretreatment with the $\alpha_{2}$-adrenergic antagonist piperoxane, whereas $\beta$-adrenergic antagonists had no effect (Cedarbaum and Aghajanian, 1976).

Aghajanian initially proposed an elegant hypothesis to explain how $\alpha_{2}$-receptors regulate the LC. This hypothesis was based on the fact that the response of LC neurons to stimulation is biphasic, consisting of short bursts of impulses followed by a period of quiescence, or post stimulation inhibition (PSI). In unanesthetized, behaving animals, this biphasic response occurs to a wide range of sensory stimuli (Foote et al., 1980; AstonJones and Bloom, 1981). In anesthetized animals, the range of sensory stimuli that trigger this biphasic response is much reduced, but still occurs to noxious peripheral stimulation (Cedarbaum and Aghajanian, 1976); additionally, clcctrical stimulation of sciatic nerve (Cedarbaum and Aghajanian, 1978) and antidromic activation of LC neurons through dorsal bundle or cingulum stimulation (Aghajanian et al., 1977) produce the characteristic burst of LC activity followed by PSI. On the basis of electrophysiological studies, as well as of anatomical data revealing that LC axons send off collateral branches within the LC (Shimizu and Imamoto, 1970; Swanson, 1976), Aghajanian and his colleagues (1977) hypothesized that when LC neurons depolarize, they release their transmitter (NE) from recurrent collaterals within the LC onto $\alpha_{2}$-receptors, which then inhibit LC activity for a brief period. That is, $\mathrm{LC}$ recurrent collaterals release NE during the "bursting" portion of the biphasic LC response, and the released NE then stimulates the $\alpha_{2}$-receptors to produce the inhibition observed following the burst. Support for this hypothesis was obtained from extracellular single-unit recording studies demonstrating that blockade of $\alpha_{2}$-receptors, by systemic or ionotophoretic administration of the $\alpha_{2}$-antagonist piperoxane, reduced the duration of the PSI following antidromic (Aghajanian et al., 1977) or orthodromic (Cedarbaum and Aghajanian, 1978) activation of LC neurons.

Elegant as this formulation was, Aghajanian and colleagues subsequently discovered empirical difficulties with the notion that $\alpha_{2}$-receptors arc rcsponsible for the PSI via transmitter release from recurrent collaterals. In performing the first intracellular recordings from LC neurons in vivo, these investigators discovered that intracellular activation of an LC neuron was capable of producing PSI (Aghajanian and VanderMaelen, 1982; Aghajanian et al., 1983). In that intracellular stimulation is likely to depolarize only the single neuron being stimulated, this meant that each LC neuron would have to have recurrent collaterals 
onto itself to be consistent with the mechanism originally suggested. Aghajanian and VanderMaelen (1982) argued that this was unlikely in all the neurons they tested and, instead, suggested that a calcium-activated increase in potassium conductance produced directly by depolarization might explain PSI. Subsequently, Andrade and Aghajanian (1984a) quantified a number of electrophysiological parameters following antidromic invasion of LC neurons, such as the relationship between PSI and interspike interval, and concluded that a calcium-activated potassium conductance was indeed primarily responsible for producing PSI. Finally, Andrade and Aghajanian (1984b) showed that PSI was not eliminated by blockade of $\alpha_{2}$-receptors in vitro. Thus, a series of results now indicate that $\alpha_{2}$-receptors are not necessary for PSI and that PSI arises directly from depolarization via a calcium-dependent potassium conductance. It should be mentioned, however, that Ennis and Aston-Jones (1986) obtained results indicating that $\alpha_{2}$-receptors may contribute to PSI.

The foregoing suggests that $\alpha_{2}$-receptors play considerably less of a role in PSI than was originally thought. Consequently, we examined the possibility that $\alpha_{2}$-receptors might influence a different aspect of LC activity than had been studied heretofore. Two observations previously made by other investigators were instrumental in suggesting a different emphasis. The first observation was that the magnitude of the neuronal response of LC neurons to both simple (tone, light flash, touch) and complex (food, novel objects) stimuli varies positively with the vigilance of the animal (Foote et al., 1980; Aston-Jones and Bloom, 1981): the more vigilant the state of the animal, the greater the magnitude of the neuronal response. This suggested to us that the initial response of LC neurons to stimulation was a particularly important aspect of LC activity. The second observation was that the dose of the highly selective $\alpha_{2}$-adrenergic antagonist idazoxan required to elevate the spontaneous firing rate of LC neurons was much higher than the dose of the same drug required to reverse (or block) the inhibition of firing produced by the $\alpha_{2}$-agonist, clonidine (Freedman and Aghajanian, 1984). Thus, a low dose of an adrenergic antagonist that appeared to be affecting $\alpha_{3}$-receptors so as to block the action of an adrenergic agonist had no effect on the firing rate of the LC, suggesting that the antagonist could be affecting the receptors in some way that was not apparent from examining the spontaneous (or baseline) rate of firing. Putting these 2 observations together suggested that onc should consider, first, whether $\alpha_{2}$-receptors influence the initial response of the LC to sensory input rather than just the baseline firing rate of the LC and, second, whether $\alpha_{2}$-blocking agents affect this initial LC response at doses below that required to elevate the basal firing of unstimulated LC. To test the hypothesis that $\alpha_{2}$-receptors modulate the responsiveness of LC neurons to stimuli, the effect of $\alpha_{2}$-receptor blockade, at doses both above and below those required to increase spontaneous LC activity, on the responses of LC neurons to excitatory stimulation was studied.

\section{Materials and Methods}

Preparation of animals. The subjects were male Sprague-Dawley rats obtained from Charles River Breeding I aboratories and weighing 250 $400 \mathrm{~g}$ at the time of experimentation. Stereotaxic surgery was performed, and extracellular recordings from the LC were obtained, under chloral hydrate anesthesia using an initial intraperitoneal dose of $400 \mathrm{mg} / \mathrm{kg}$ with supplemental doses administered through a lateral tail vein as needed. Once the incisor bar was adjusted so that both bregma and lambda suture landmarks lay in the same horizontal plane (skull flat), a burr hole was made $1.1 \mathrm{~mm}$ posterior to lambda and $1.1 \mathrm{~mm}$ lateral to the sagittal suture.

Electrophysiological recordings. Two types of electrodes were used. Single-barrel glass electrodes were produced by pulling Omega Dot micropipettes ( $1.5 \mathrm{~mm}$ outside diameter, Frederick Haer) and filling them with $2 \mathrm{M} \mathrm{NaCl}$ saturated with Fast green. The tips were then broken back to obtain electrode impedances of 3-5 M 2 measured in saline at $135 \mathrm{~Hz}$. Insulated steel microelectrodes (Frederick Haer, $5 \mu \mathrm{m}$ exposed tip length, 2-5 M 2 ) were also employed in the experiments requiring infusion of drug directly into the $\mathrm{LC}$, for which an extremely thin recording electrode was desirable. The action potentials from spontaneously firing, single units were amplified and displayed on a Tektronix $5111 \mathrm{~A}$ oscilloscope. Individual spikes were isolated, then integrated over either 1 or $10 \mathrm{sec}$ periods by an electronic counter. The number of spikes occurring over consecutive periods was recorded in 2 ways: The analog output of the counter was graphically recorded, and the digital output of the counter was recorded on a thermal printer. In addition, signals from the amplifier drove an audiomonitor.

The LC neurons were found at a depth of $5.5-6.5 \mathrm{~mm}$ below the dura at the coordinates for the burr hole presented above. All cells fired at a regular low rate $(0.5-3.5 / \mathrm{sec})$ and were observed to respond to noxious stimulation (e.g., pressure) applied to the contralateral hind paw with a burst of firing followed by a prolonged quiescent period. Each LC spike exhibited a positive-negative waveform with a notch on the ascending limb. Additional landmarks helpful in locating LC cells included (1) a zone of relative silence just ventral to the cerebellum and dorsal to the LC, corresponding to the fourth ventricle; (2) an area of highfrequency activity just lateral to the LC that responded with a burst of activity to displacement of the lower jaw, corresponding to the mesencephalic nucleus of the fifth nerve; (3) a zone of low activity just medial to the LC, corresponding to the central gray; and (4) a zone of high-frequency, rhythmic activity ventral to the LC, corresponding to the reticular formation. Histological verification of glass electrode placements was obtained by passing $20 \mu \mathrm{A}$ of negative-going current through the recording electrode for $45 \mathrm{sec}$, thereby depositing a spot of Fast green at the electrode tip. The brains were removed, imbedded in paraffin, and cut in $10 \mu \mathrm{m}$ sections; the sections were then mounted and stained with cresyl violet.

Drug administration. Pharmacological manipulations were achieved with intravenous drug administration through a lateral tail vein or through direct infusion of drug into the LC. In the latter method, the drug was infused into the LC via a cannula mounted adjacent to the recording electrode. This cannula was made of 32 gauge stainless steel hypodermic tubing, which was cemented next to the steel recording electrode. The tip of the recording electrode protruded $250 \mu \mathrm{m}$ below the tip of the cannula, a configuration that was found to produce stable electrophysiological recordings without sacrificing cannulae proximity to the neuron being recorded. Approximately $2 \mathrm{~cm}$ above the cannula tip, the stainless steel tubing was bent at a $30^{\circ}$ angle; thus, the electrode could be securely mounted in the stereotaxic carriage with the cannula deflected to the side of the electrode mount. The cannula was attached by an airtight system to a motor-driven microsyringe. This system consisted of tubing leading from the 32 gauge stainless steel cannula to a $10 \mu \mathrm{l}$ microsyringe held in a Delsaga microdoser syringe driver; this system was capable of delivering small amounts (as little as $0.05 \mu 1$ ) of fluid at uniform speed through the cannula. Immediately prior to lowering of the recording electrode and cannula assembly, the substance to be infused was advanced to the tip of the cannula, after which the plunger of the infusion syringe was slightly withdrawn to create a small (approximately $1.0 \mathrm{~mm}$ ) deadspace in the end of the cannula that prevented drug from leaching out of the cannula tip before injection was to be made.

Drugs employed were idazoxan hydrochloride (Reckitt and Colman, Kingston-upon-Hull, England), nicotine sulfate (Sigma Chemical Company), and yohimbine hydrochloride (Sigma). All drugs were administered in $0.9 \% \mathrm{NaCl}$. If a supplemental dose of chloral hydrate was required, testing was always delayed at least $5 \mathrm{~min}$ following administration of the anesthetic.

Testing procedure. Compression of the contralateral hind paw produces a transient burst of LC neuronal activity that is followed by a quiescent period. Typically used in electrophysiological studies as a preliminary means of demonstrating that an electrode is recording from within the LC, this manipulation served as an cxcitatory stimulus in 3 of the 4 experiments to be described. Repeated compressions of similar magnitude were produced by placing the contralateral hind paw between a pair of surgical forceps; the experimenter then applied enough pressure 


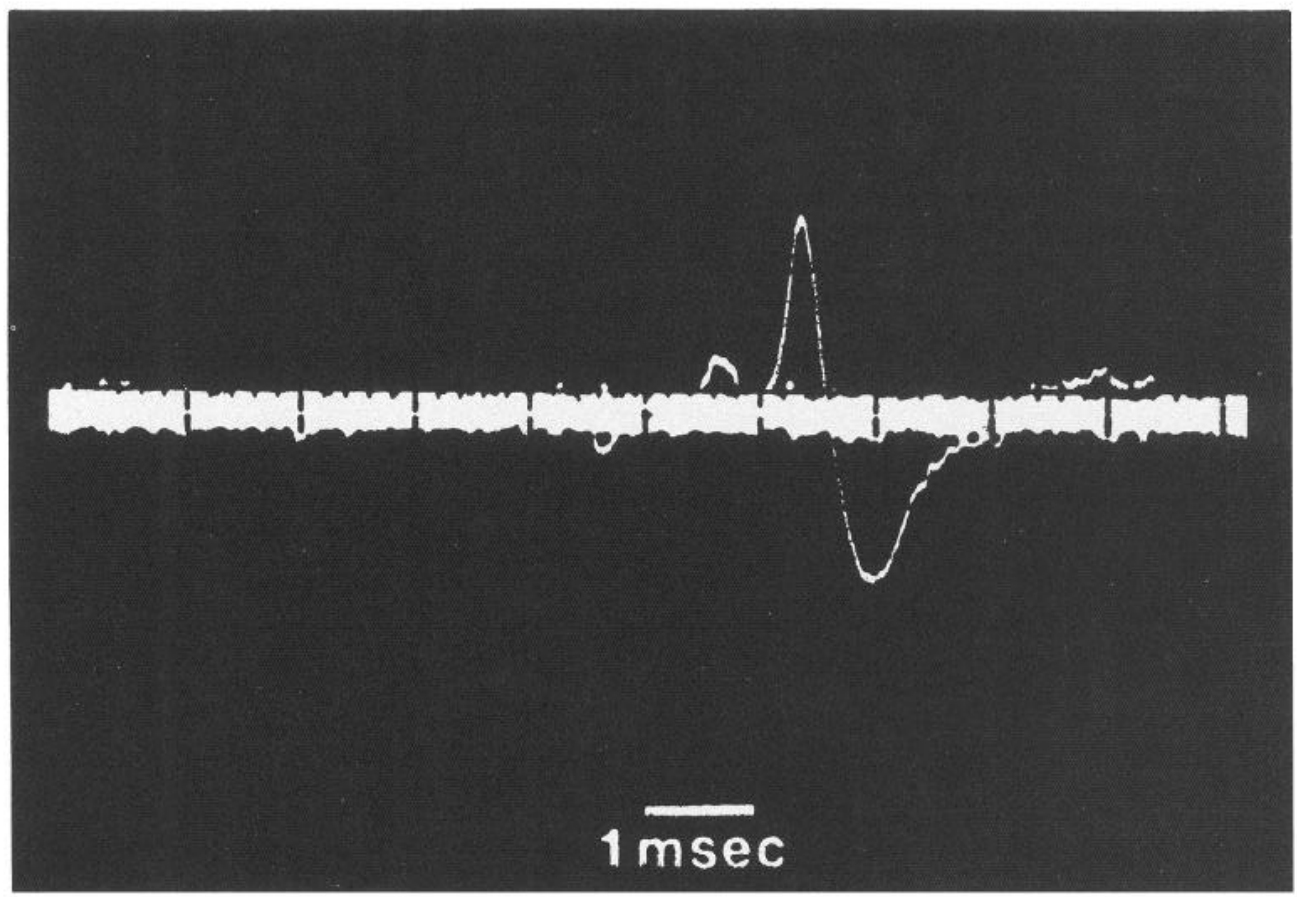

Figure 1. Top, Typical positive-negative waveform of a spontaneously firing LC neuron. Note the notch on the ascending limb. Bottom, Typical response pattern of a spontaneously firing LC neuron to the brief application of a noxious stimulus in the form of contralateral hind paw compression $(P C)$. All recorded cells fired at a slow, regular rate $(0.5-3.5 \mathrm{~Hz})$. Note the increase in firing rate with stimulus onset, followed by a prolonged quiescent period after stimulus offset.

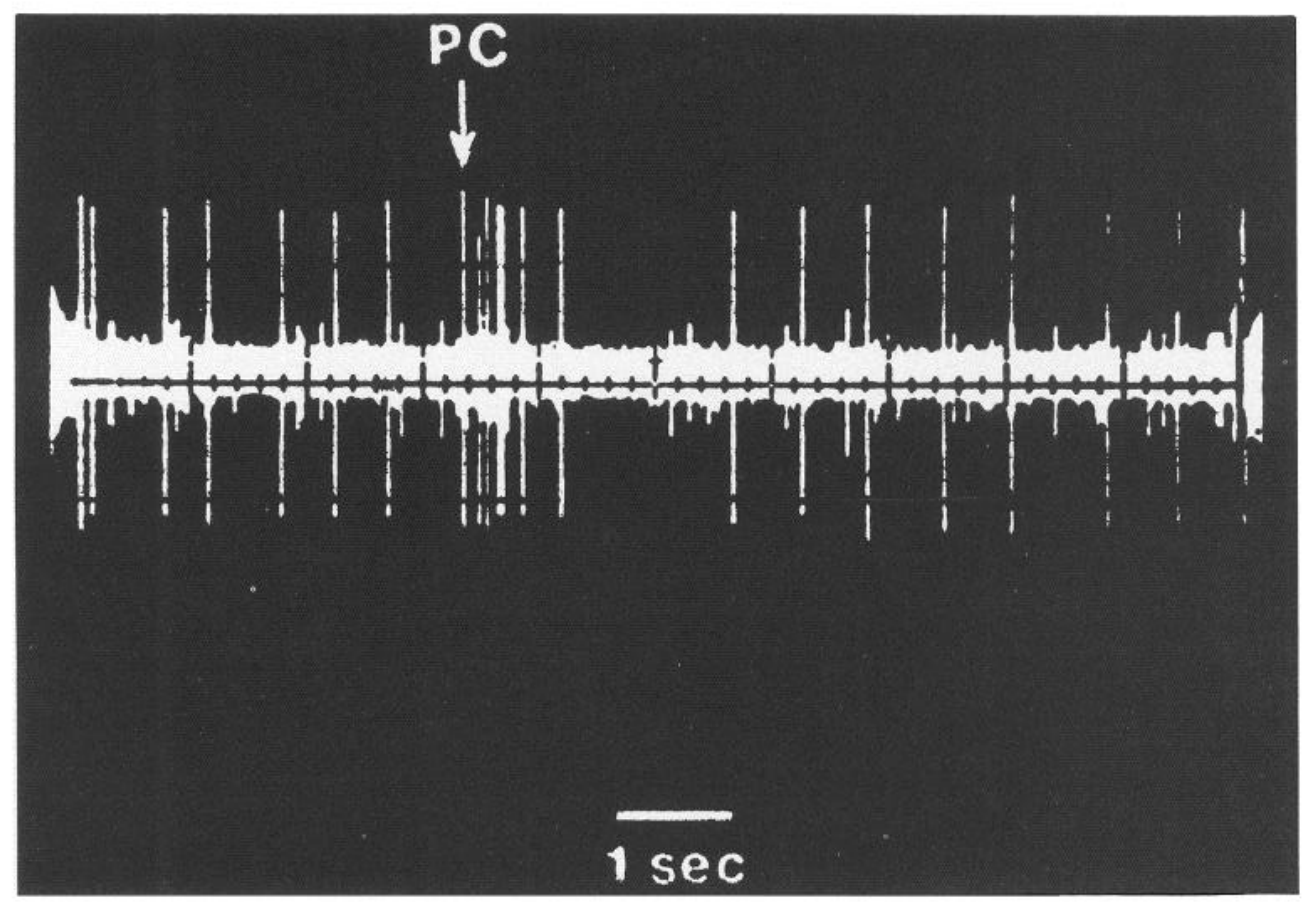

midway along the forceps to bring opposite sides of the forceps, at this midway point, into contact. Compressions of similar duration $(1 \mathrm{sec})$ were produced with the aid of a digitally controlled timer. This electronic timer signaled the experimenter to commence compression by emitting a tone in conjunction with an electronic flash. The combined auditory/ visual signal was repeated at the end of the $1 \mathrm{sec}$ interval, the onset of which signaled the experimenter to release pressure from the forceps.

The experiments utilizing this contralateral hind paw compression (PC) procedure shared a common paradigm. In these experiments, an LC cell was isolated and a baseline rate determined by recording spon- taneous activity for a minimum of $5 \mathrm{~min}$. While baseline was being determined, spikes were integrated over $10 \mathrm{sec}$ periods. The responsiveness of the cell to PC was then determined by performing 10 trials consisting of $1 \mathrm{sec}$ PC with $10 \mathrm{sec}$ intervals between compressions. During these trials, the number of spikes occurring over each $1 \mathrm{sec}$ epoch was recorded. An average firing rate was calculated based on the 10 rates obtained during each $1 \mathrm{sec}$ PC. Two minutes after completion of these trials, a drug manipulation was performed (by intravenous or direct LC administration). In the first study, groups of rats received various doses of idazoxan intravenously; in the second study, groups 


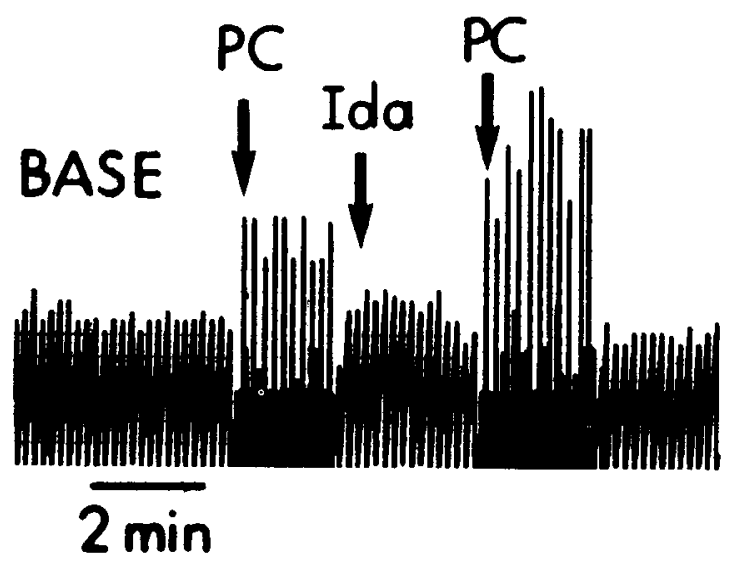

Figure 2. Strip-chart record showing the augmentation by the $\alpha_{2}$-adrenergic antagonist idazoxan $(25 \mu \mathrm{g} / \mathrm{kg}$, i.v.) of the response to paw compression $(P C)$ of an LC neuron in a typical subject. After a baseline rate of activity was established, the magnitude of the response of the LC neuron to PC was recorded on 10 trials. Idazoxan (Ida) was then administered, and the postdrug neuronal response to PC tested. This procedure was repeated in 4 subjects at each of the doses of idazoxan employed (see Fig. 3).

received varying doses of idazoxan by infusion; and in the third study, a group received yohimbine intravenously. A postdrug baseline rate was then determined by again integrating spikes over $10 \mathrm{sec}$ periods for 3 min, with the last minute determining the base rate. Finally, the responsiveness of the LC cell to PC in the presence of drug was determined by repeating the 10 trials of $1 \mathrm{sec} P C$ with $10 \mathrm{sec}$ intertrial intervals. Again, the average of the 10 rates obtained during PC was calculated.

The fourth study employed intravenously administered idazoxan, but nicotine sulfate $(25 \mathrm{mg}$, i.v.) replaced $\mathrm{PC}$ as the means for transiently increasing LC activity. After determining the base rate of spontaneous activity, the responsiveness of the cell to nicotine was determined by intravenously administering the drug, then integrating spikes over 10 sec periods for $7 \mathrm{~min}$. This amount of time allowed the cell to respond to $25 \mathrm{mg} / \mathrm{kg}$ nicotine and then return to its previous level of baseline activity. The procedure was then repeated: A second injection of nicotine was administered and the response recorded following the same procedure as with the first injection. This second trial of nicotine tested for the possibility that the drug might have larger effects based simply on a second administration. After completion of these trials, a drug manipulation was performed: Idazoxan was intravenously administered and a postdrug baseline rate again determined by integrating spikes over $10 \mathrm{sec}$ periods for $3 \mathrm{~min}$. Finally, the responsiveness of the neuron to nicotine in the presence of idazoxan was determined by repeating the nicotine procedure described above, carrying out at least 2 nicotine injections.

Statistical analysis. Activity of the LC (depolarizations/sec) was analyzed by analysis of variance. Since the same subjects were used for all measurements of a given dose of drug, a repeated-measures analysis of variance (subjects $\times$ conditions) was carried out at each dose. In the event that a significant overall $F$ was obtained at a given dose, comparisons of effects at that dose (e.g., baseline predrug vs. baseline postdrug or PC predrug vs. PC postdrug) were then carried out by protected $t$ tests.

\section{Results}

Recordings were made from a total of $51 \mathrm{LC}$ neurons in an equal number of rats. All LC neurons displayed a similar waveform (Fig. 1, top) and responded to $\mathrm{PC}$ with an increase in firing rate that was followed by a period of quiescence (Fig. 1, bottom).

\section{Intravenously administered idazoxan increases the responsiveness of $L C$ neurons to noxious stimulation}

Twenty-eight LC neurons ( 1 neuron in each of 28 rats) were recorded from in this phase of the study. Six groups of 4 rats received a single dose of idazoxan through a lateral tail vein. The doses ( $\mu \mathrm{g}$ of drug $/ \mathrm{kg}$ body weight) were as follows: 10,18 , $25,60,140$, and 640 . A control group received $0.9 \%$ saline through the lateral tail vein. Idazoxan solutions were such that all animals were administered volumes ranging from 0.05 to $0.15 \mathrm{ml}$.

As shown in Figures 2 and 3, intravenous administration of the $\alpha_{2}$-adrenergic antagonist idazoxan markedly increased responsiveness of LC neurons to $\mathrm{PC}$ at doses far below those required to alter baseline activity. Although baseline rates were

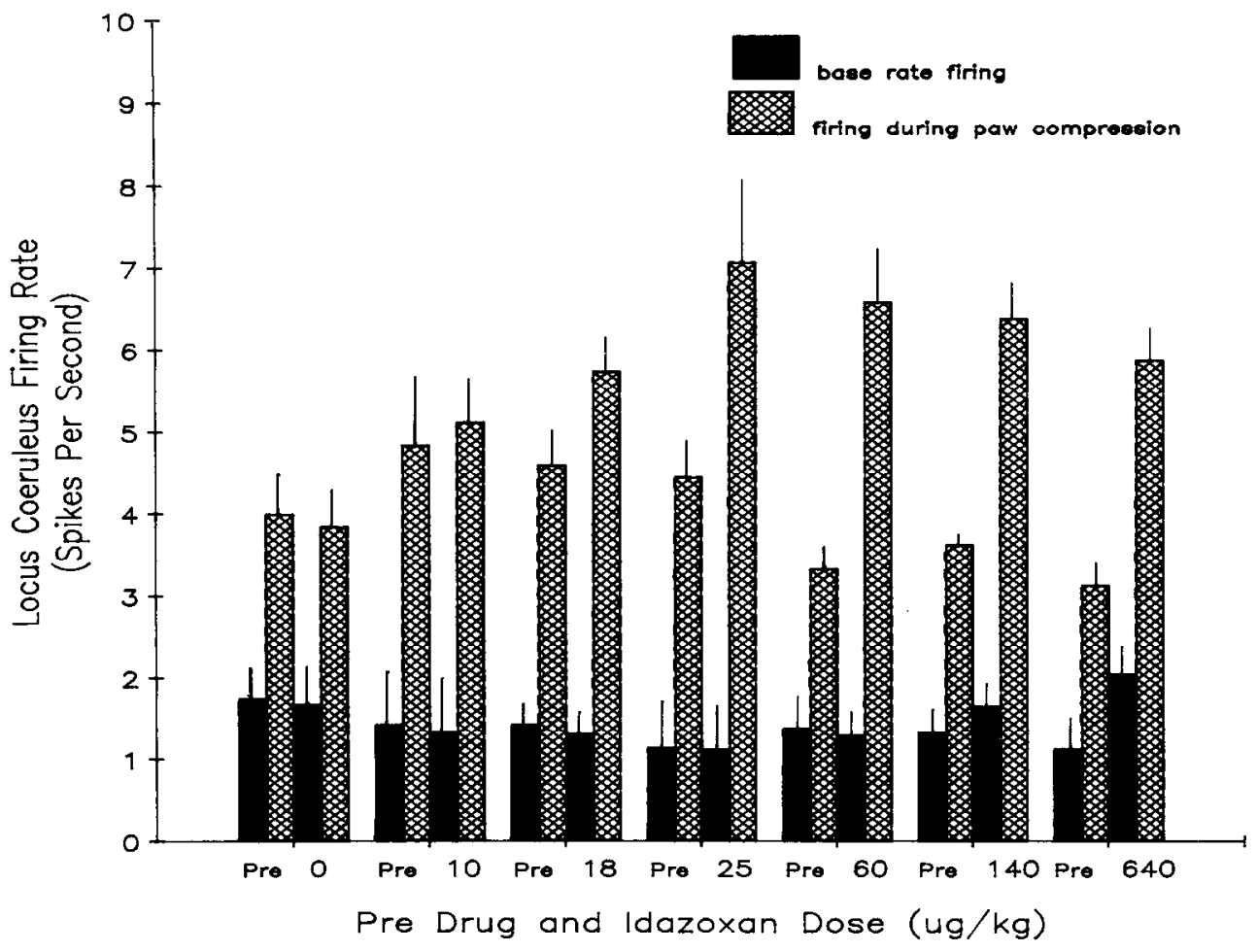

Figure 3. Increase in the responsiveness of locus coeruleus neurons to contralateral hind paw compression (PC) by idazoxan. Filled bars indicate spontaneous firing rates of LC neurons, while hatched bars indicate firing rates during the application of PC. After a predrug baseline firing rate and predrug response to PC rate were determined (Pre), idazoxan was administered intravenously (dose indicated on graph), and baseline firing rate and response to $\mathrm{PC}$ were again measured. Each dosage of idazoxan was administered to 4 animals, and no animal received more than 1 dose. Note that although baseline activity was not increased until $640 \mu \mathrm{g} /$ $\mathrm{kg}$ idazoxan was administered, there was a significantly larger response to PC following $18 \mu \mathrm{g} / \mathrm{kg}$ idazoxan. 
Figure 4. LC firing rates during the first second following termination of PC. Filled bars indicate spontaneous firing rates, while hatched bars indicate the firing of the LC neuron during the $1 \mathrm{sec}$ period immediately following the offset of PC. Although a poststimulation quiescent interval can be observed in both the predrug and idazoxan conditions, there is significantly greater poststimulation activity following idazoxan treatment beginning with doses of $25 \mu \mathrm{g} / \mathrm{kg}$.

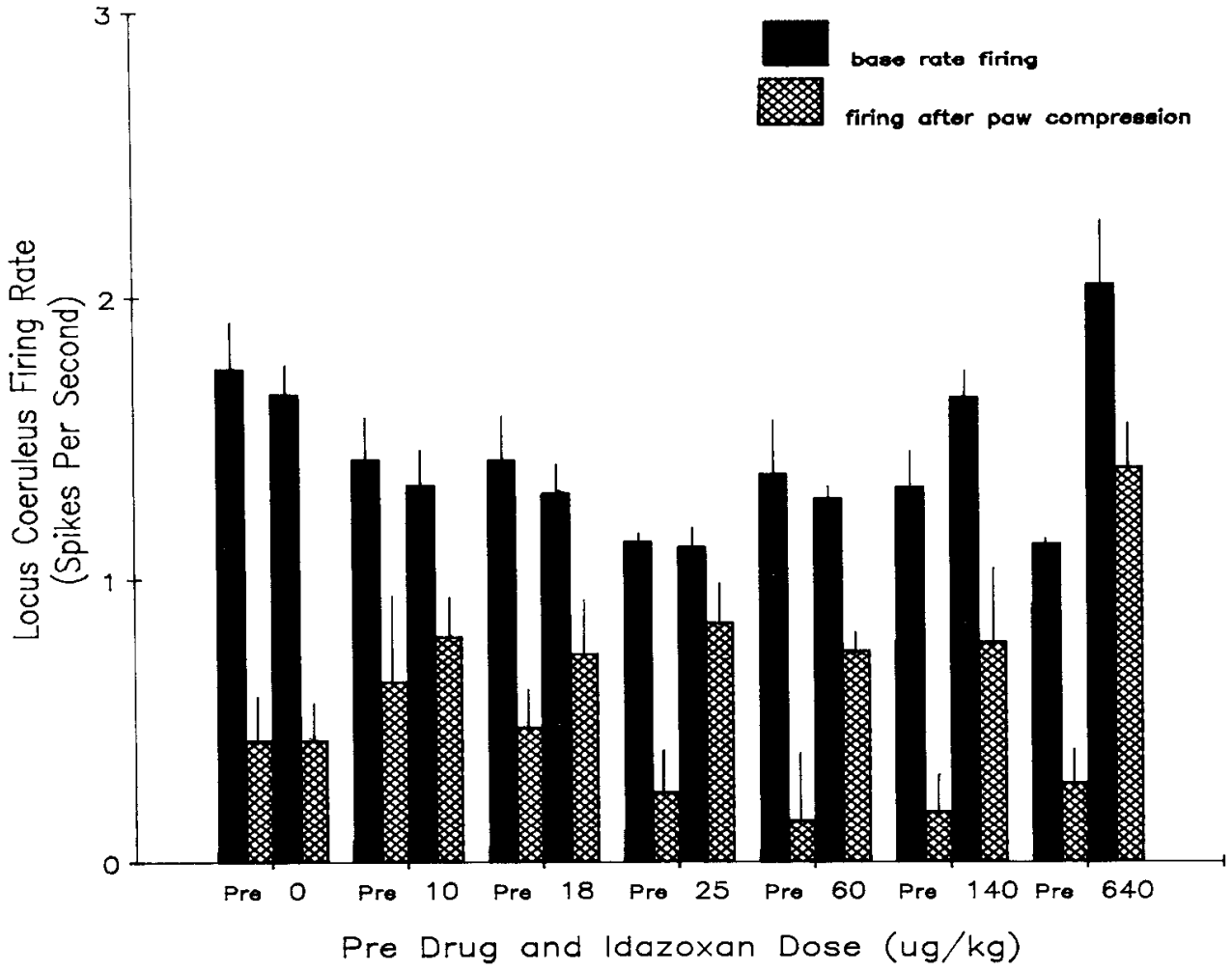

not significantly increased until doses of $640 \mu \mathrm{g} / \mathrm{kg}$ were reached $(t=4.79 ; p<0.01)$, the rcsponsc to PC was significantly increased following idazoxan administration beginning with doses as low as $18 \mu \mathrm{g} / \mathrm{kg}[t(9 d f)=2.09 ; p<0.05]$. The increased response to $\mathrm{PC}$ was seen in every animal given a dose above 18 $\mu \mathrm{g} / \mathrm{kg}$ despite the fact that animals varied widely in the responsiveness of $\mathrm{LC}$ neurons to $\mathrm{PC}$ in the predrug condition. Interestingly, the magnitude of increase in LC firing produced by $\mathrm{PC}$ did not correlate with the baseline activity rate either before the drug was given (correlation $r=0.08 ; t=0.41$ ) or after (correlation $r=0.16 ; t=0.83$ ).

LC firing rates obtained during the first sec following the termination of $\mathrm{PC}$ are examined in Figure 4. A poststimulation quiescent interval was clearly observed in both the predrug and idazoxan conditions. However, Figure 4 shows that poststimulation activity was increased following idazoxan treatment beginning with doses of $25 \mu \mathrm{g} / \mathrm{kg}[t(9 d f)=2.97 ; p<0.01]$. By 2 sec after PC termination, LC activity in both predrug and idazoxan conditions had essentially returned to the baseline firing rate (Fig. 5).

\section{Infused idazoxan increases the responsiveness of $L C$ neurons to noxious stimulation}

The results described above demonstrate that the $\alpha_{2}$-antagonist idazoxan can increase the responsiveness of the LC to noxious stimulation and that this occurs at doses below those that alter baseline firing rate of LC neurons. It could be argued, however, that the systemically administered idazoxan exerted its effects not by increasing the responsiveness of the $\mathrm{LC}$ to $\mathrm{PC}$, but rather by acting at other central or perhaps even peripheral sites to increase the amount of stimulation received by the $\mathrm{LC}$ during PC. To determine whether this might be so, an experiment was performed in which small amounts of idazoxan were infused directly into the region from which the electrode was recording. Should an increase in the responsiveness to $\mathrm{PC}$ be found in this case, the results would lend support to the notion that idazoxan was acting directly at the $\mathrm{LC}$ to increase $\mathrm{LC}$ responsiveness to noxious stimulation.

Twelve LC neurons ( 1 neuron in each of 12 rats) were recorded from in this study. Two groups of 4 rats received idazoxan through a cannula mounted adjacent to the recording electrode. The first group received $1.0 \mathrm{ng}$ of idazoxan, while the second group received $22.0 \mathrm{ng}$. A third group received $0.9 \%$ saline via a cannula. All volumes were $1.0 \mu \mathrm{l}$ delivered over $15 \mathrm{sec}$. In addition, 2 animals from the first group received increasing doses of idazoxan. As shown in Figure 6, infusion of idazoxan into the $\mathrm{LC}$ increased the response to $\mathrm{PC}$ in both groups given the drug $[t(9 d f)=7.74 ; p<0.001$, for $1.0 \mathrm{ng}$ infusion group; $t(9 d f)=3.51, p<0.005$ for $22.0 \mathrm{ng}$ infusion group] without having significant effects on baseline rates. This can also be seen in the 2 animals given multiple idazoxan injections (Fig. 7), where it will be observed that idazoxan progressively increased the responsiveness to $\mathrm{PC}$ while producing little increase in baseline rate.

\section{Yohimbine increases the responsiveness of $L C$ neurons to noxious stimulation}

To determine whether the effects presented in the 2 studies above were unique to the particular $\alpha_{2}$-antagonist employed, another $\alpha_{2}$-antagonist, yohimbine, was intravenously administered to 5 animals at a dose of $0.25 \mathrm{mg} / \mathrm{kg}$. As with idazoxan, the $\alpha_{2}$-antagonist yohimbine significantly potentiated the response of LC neurons to $\mathrm{PC}[t(12 d f)=2.68 ; p<0.01]$ without increasing baseline rates $[t(12 d f)=0.19]$ (see Fig. 8). 


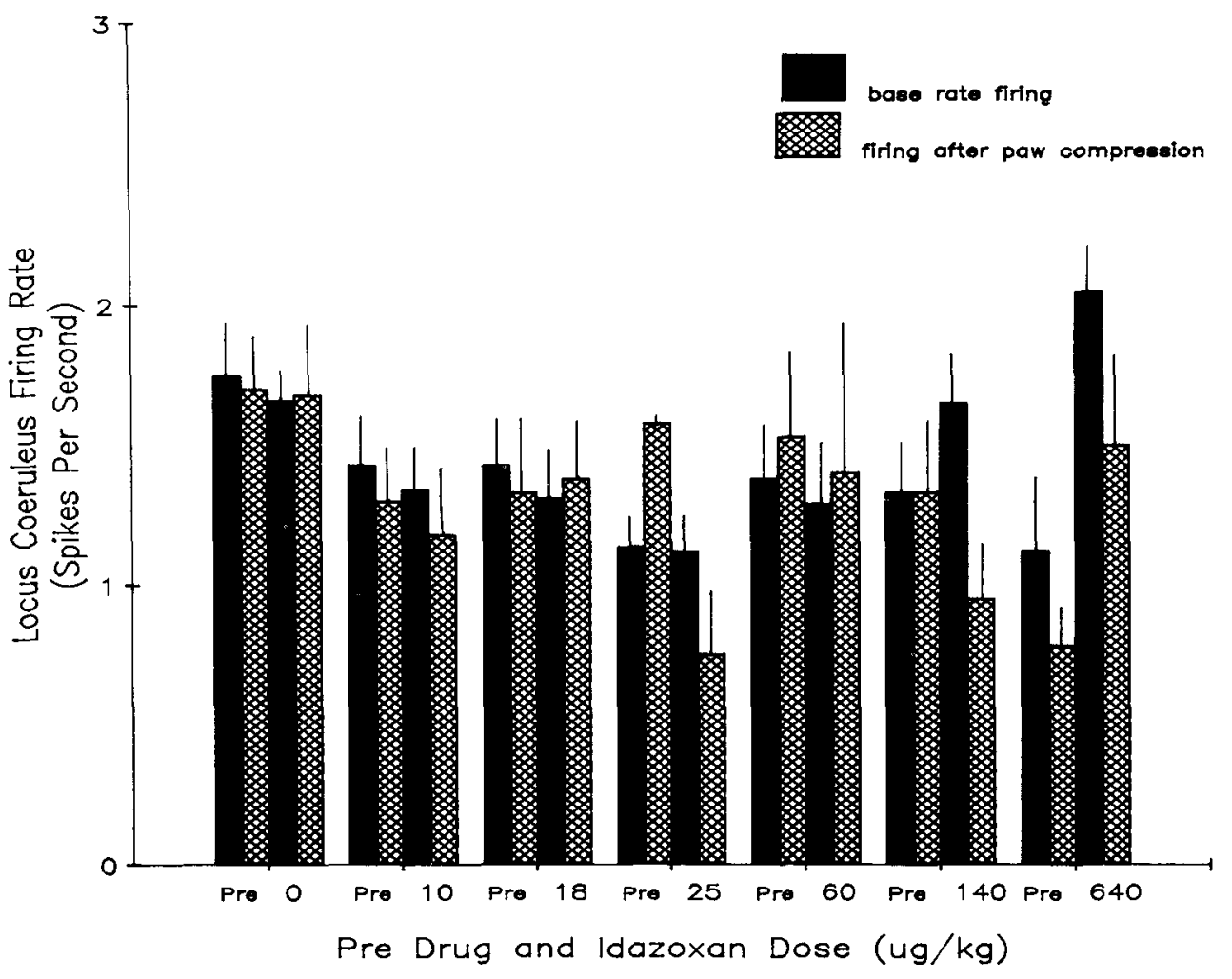

Figure 5. LC firing rates during the second second following the termination of PC. Filled bars indicate spontaneous firing rates, while hatched hars indicate the firing of the LC neuron during the second second following PC offset. Both the pre- and postdrug conditions essentially recovered from poststimulation quiesence.
Idazoxan increases the responsiveness of $L C$ neurons to nicotine

To determine whether $\alpha_{2}$-receptor blockade would augment the response of LC neurons to excitatory input other than that produced by noxious peripheral stimulation, nicotine was given instead of PC. Nicotine was substituted for PC because (1) it has been shown to transiently increase LC single-unit firing rates (Engberg and Svensson, 1980), and (2) it offered a different modality of stimulation than noxious cutaneous stimulation.

Six LC neurons were recorded in 6 rats (i.e., 1 cell/ rat). As shown in Figures 9 and 10, injections of nicotine $(25 \mathrm{mg} / \mathrm{kg}$,

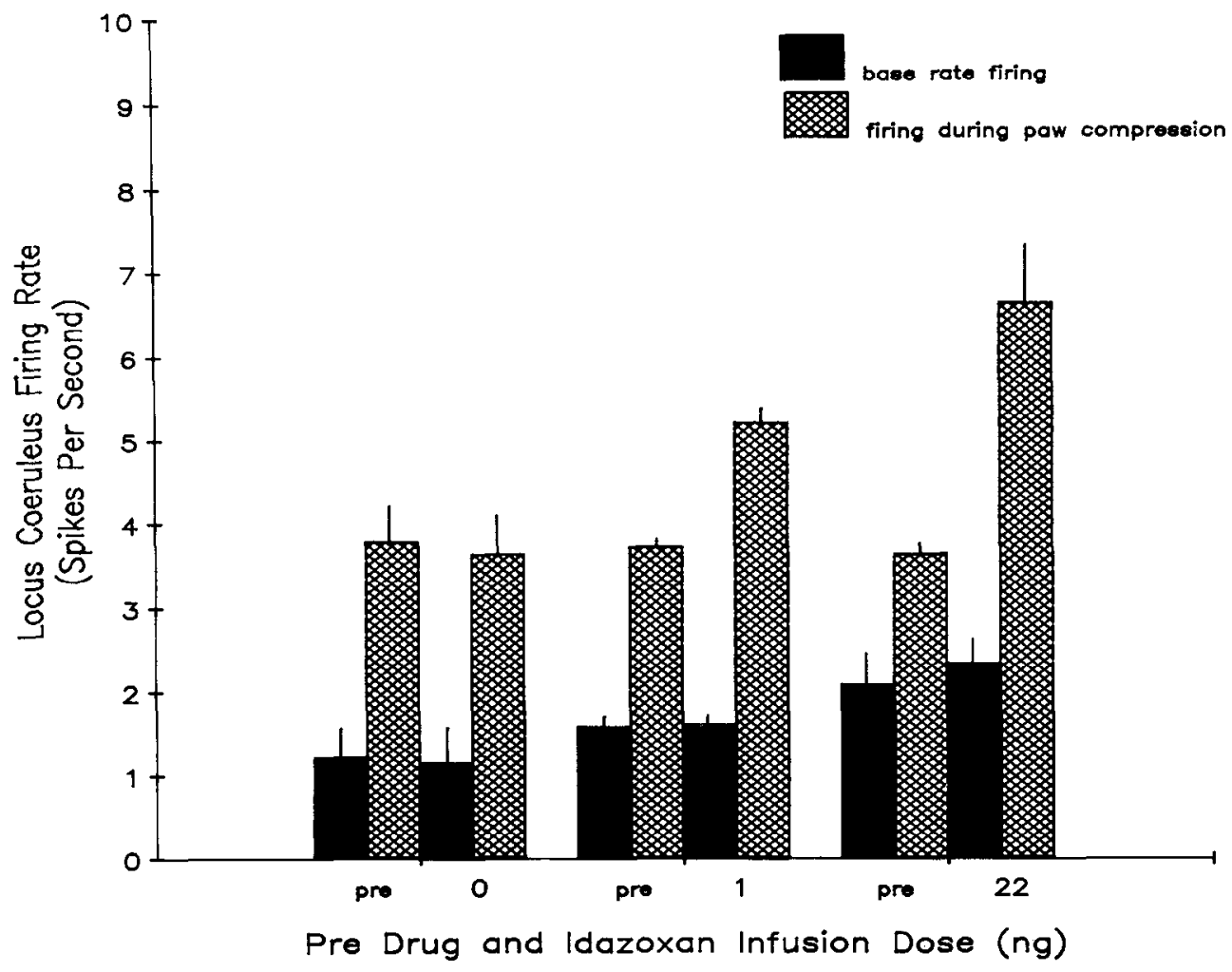

Figure 6. Augmentation of the responses of locus coeruleus neurons to paw compression (PC) resulting from direct infusion of idazoxan into the LC. Filled bars indicate spontaneous firing rates of $\mathrm{LC}$ neurons, while hatched bars indicate firing rates during the application of PC. Idazoxan was infused directly into the $\mathrm{LC}$ after a predrug baseline firing rate and predrug response to $P C$ rate were determined. Each dosage of idazoxan was administered to 4 animals, and no animal received more than 1 dose. Note that there was an increase in $\mathrm{LC}$ responsiveness to $\mathrm{PC}$ following infusion of just $1.0 \mathrm{ng}$ idazoxan, which represents approximately $1 / 10,000$ the smallest intravenous dose required for a similar effect (Fig. 3). 
Figure 7. Dose-response curve showing the augmentation of the responses of LC neurons to paw compression (PC) resulting from direct infusion of idazoxan into the $\mathrm{LC}$ in 2 animals. Filled bars indicate spontaneous firing rates of LC neurons, while hatched bars indicate firing rates during the application of PC.

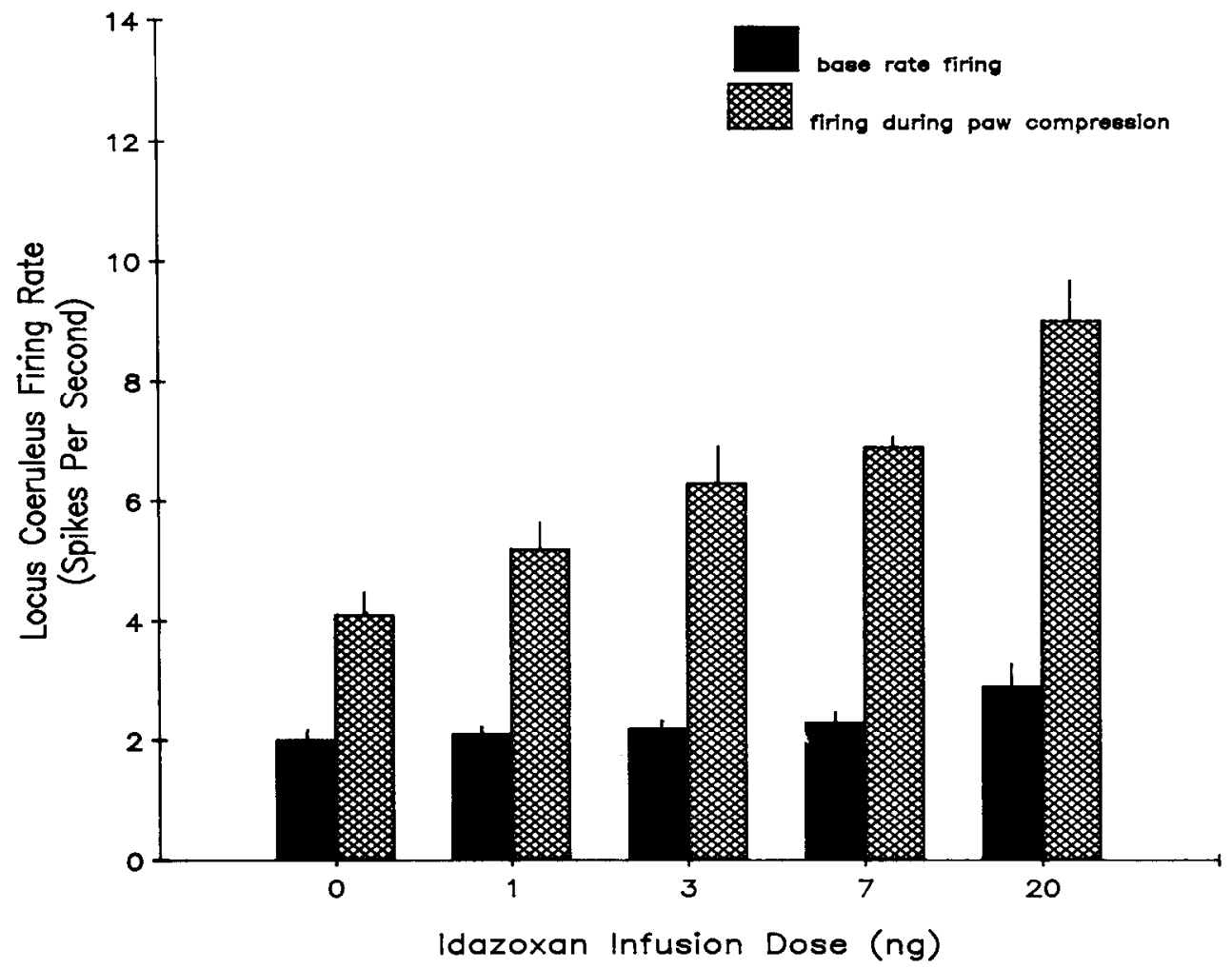

i.v.) transiently increased the firing rate of LC neurons. This effect on LC neuronal activity typically reached a maximum within 1 min of injection, with LC activity returning to baseline (i.e., pre-nicotine) levels within $7 \mathrm{~min}$. A second administration of nicotine $(25 \mathrm{mg} / \mathrm{kg}$, i.v.) showed similar effects on LC activity. After idazoxan $(140 \mu \mathrm{g} / \mathrm{kg}$, i.v. ) treatment, however, nicotine's effects on LC activity were markedly increased. At the dose used, idazoxan increased the response to nicotine $[t(15 d f)=$ $2.91, p<0.01]$ without affecting baseline activity of the LC $[t(15 d f)=0.51]$.

\section{Discussion}

The major finding of the work described above is that $\alpha_{2}$-adrenergic antagonists markedly augment the responsiveness of
Figure 8. Increase in the responsiveness of $\mathrm{LC}$ neurons to contralateral hind paw compression (PC) by the $\alpha$-adrenergic antagonist yohimbine. Filled bars indicate spontaneous firing rates of LC neurons, while hatched bars indicate firing rates during the application of PC. Yohimbine was administered intravenously after a predrug baseline firing rate and predrug response to $\mathrm{PC}$ rate were determined. Yohimbine $(0.25 \mathrm{mg} /$ $\mathrm{kg}$, i.v.) was administered to 5 animals. Although baseline activity was not increased by yohimbine at this dose, there was a significantly larger postdrug response to $\mathrm{PC}$.

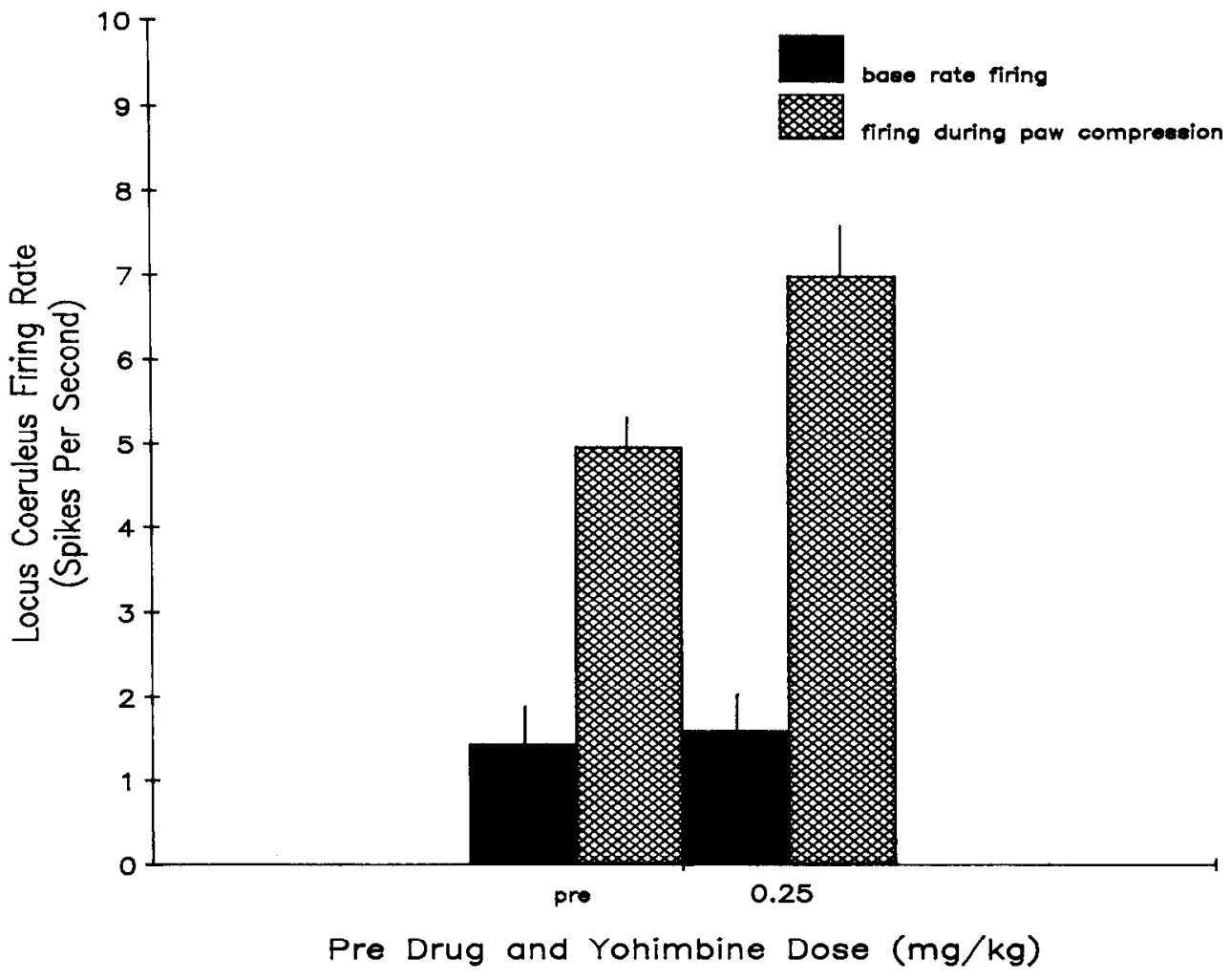




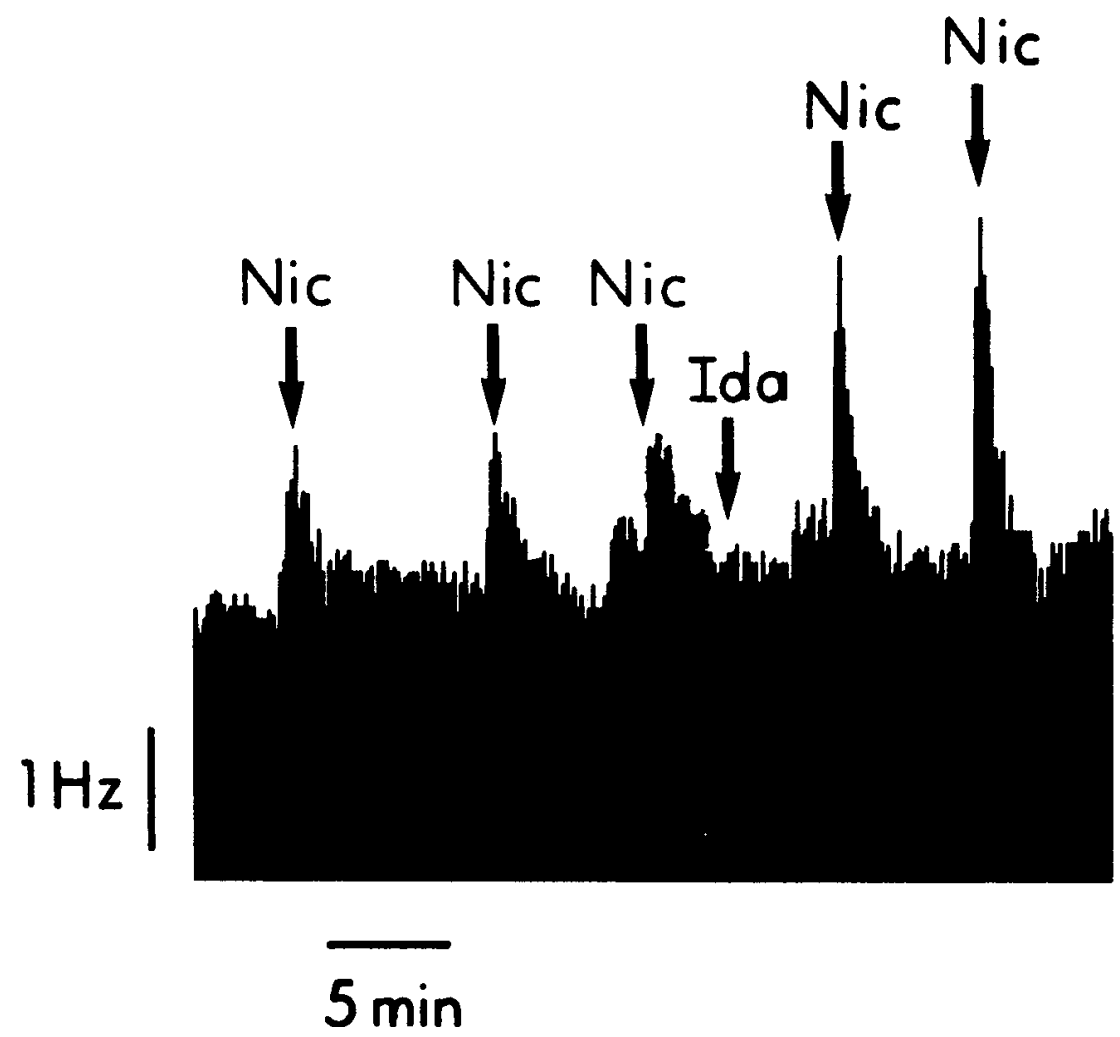

Figure 9. Strip-chart record showing the augmentation by idazoxan $(140 \mu \mathrm{g} /$ $\mathrm{kg}, \mathrm{i} . \mathrm{v}$.) of the response to nicotine injection $(25 \mathrm{mg} / \mathrm{kg}$, i.v.) of an LC neuron in a typical subject. After a baseline rate of activity was established, the magnitude of the response of the LC neuron to nicotine injection was recorded for 7 min. Following idazoxan administration, the postdrug neuronal response to nicotine injection was tested.

LC neurons to excitatory stimulation at doses far below those required to increase spontaneous activity. This finding suggests that $\alpha_{2}$-receptors in the LC play an important role in modulating the responsiveness of $L C$ neurons. The fact that idazoxan augmented the response of LC neurons to paw compression when administered directly into the LC as well as intravenously argues against the notion that idazoxan achieved this effect by increasing the amount of excitatory stimulation received by I.C. neurons. Indeed, increases in the responsiveness of $\mathrm{LC}$ neurons to paw compression by infusion of idazoxan into LC occurred at

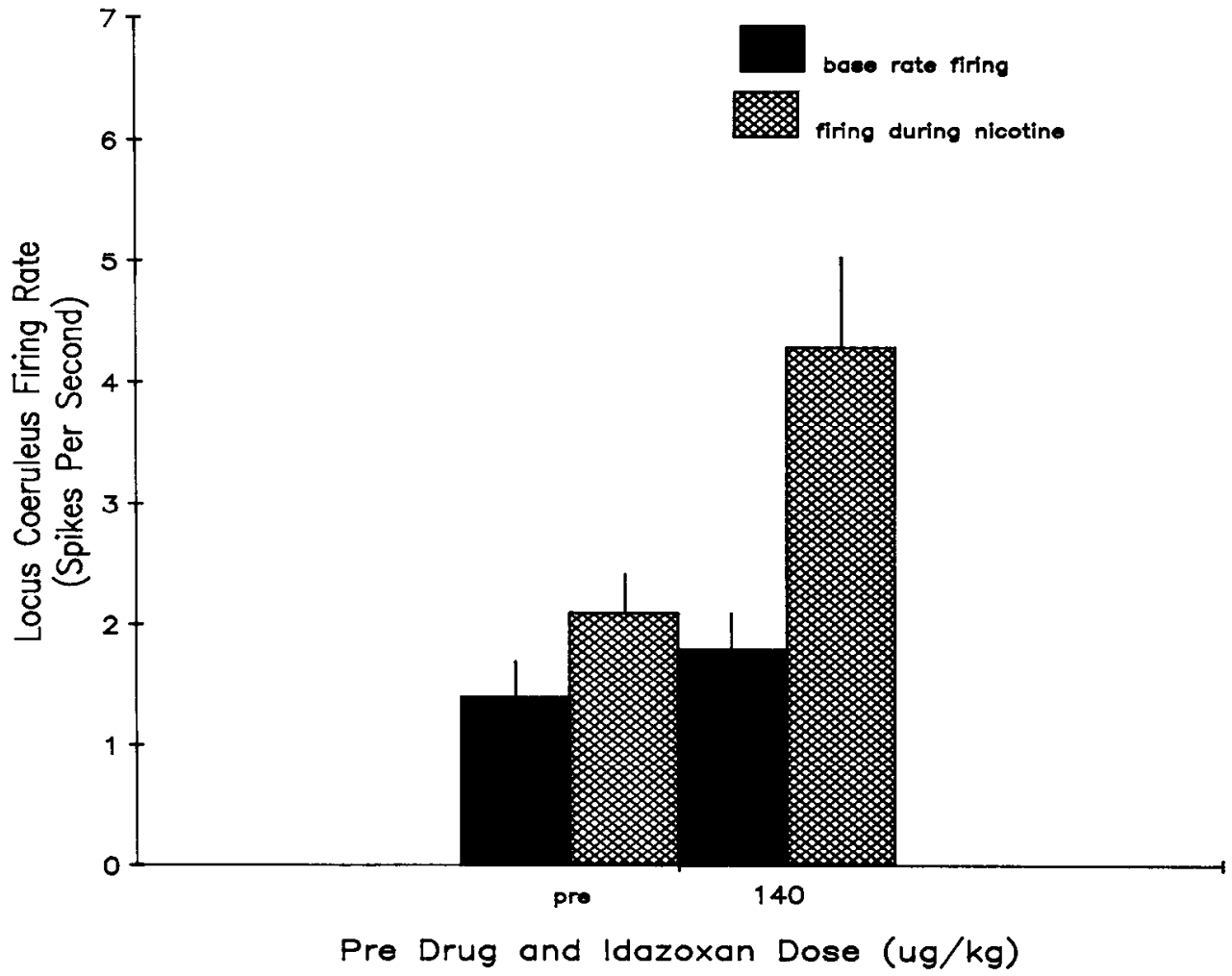

Figure 10. Augmentation of the responses of LC neurons to nicotine injection $(25 \mathrm{mg} / \mathrm{kg}$, i.v.) following idazoxan $(140 \mu \mathrm{g} / \mathrm{kg}$, i.v.). Filled bars indicate spontaneous firing rates of LC neurons, while hatched bars indicate firing rates during the first minute following the nicotine injection. Idazoxan was administered after a predrug baseline firing rate and predrug response to nicotine injection were determined. Idazoxan (140 $\mu \mathrm{g} / \mathrm{kg}$, i.v.) was administered to 6 animals. Note that although baseline activity was not increased by idazoxan, there was a significantly larger response to nicotine injection following idazoxan treatment. 
doses as low as $1 / 10,000$ of the smallest systemic dose needed to show a similar effect. Thus, it is unlikely that idazoxan influenced the LC by affecting receptors outside the LC to cause a different amount of input to the nucleus. That yohimbine as well as idazoxan was capable of increasing $L C$ responsiveness without altering spontaneous activity showed that the effects of $\alpha_{2}$-receptor blockade on $\mathrm{LC}$ responsiveness are not unique to a particular $\alpha_{2}$-adrenergic antagonist. Finally, the fact that the response of $L C$ neurons to nicotine injection as well as noxious peripheral stimulation was potentiated by idazoxan demonstrated that the effects of blockade of $\alpha_{2}$-receptors on LC responsiveness are not limited to a specific modality of stimulation.

The finding that low doses of $\alpha_{2}$-adrenergic antagonists affect the initial responsiveness of LC neurons to stimulation without altering spontaneous activity fits well with data indicating that the $L C$ appears to be particularly responsive to variations in the environment rather than steady states or repetitive events. That is, LC ncurons cxhibit biphasic responses to a varicty of sensory stimuli (Foote et al., 1980; Aston-Jones and Bloom, 1981), and respond to continuous noxious input with a burst of activity that gradually returns to near baseline levels (Cedarbaum and Aghajanian, 1978). If, as these studies suggest, an important function of the LC is to signal environmental change, then examining the effects of pharmacological substances on LC activity may be most appropriate when those neurons are concurrently activated by sensory stimuli. As shown in the present experiments, the application of paw compression revealed effects of one type of pharmacological manipulation ( $\alpha_{2}$-blockade) on LC activity at doses of the receptor blockers that would not have appeared to have had an effect had analysis been limited to measurement of spontaneous activity.

As did Aghajanian and his colleagues (Aghajanian et al., 1977; Cedarbaum and Aghajanian, 1978), we observed that blockade of $\alpha_{2}$-receptors decreased the PSI that follows a burst of firing of LC neurons (see Fig. 6). However, in the present study we noted that this decrease in the amount of postactivation inhibition was preceded by a large increase in the response of LC neurons. This raises the possibility that increased responsiveness of LC neurons accounts for the reduced PSI that Aghajanian and his colleagues observed following $\alpha_{2}$-blockade, or, in other words, that reduced PSI following $\alpha_{2}$-receptor blockade is a consequence of increased responsiveness of $\mathrm{LC}$ neurons. This explanation for reduced PSI seen following $\alpha_{2}$-blockade would fit with recent evidence suggesting that $\alpha_{2}$-receptors are less involved in PSI than was originally thought to be the case (Andrade and Aghajanian, 1984a, b; Ennis and Aston-Jones, 1986).

\section{References}

Aghajanian, G. K., and C. P. VanderMaelen (1982) Alpha-2-adrenoreceptor-mediated hyperpolarization of locus coeruleus neurons: Intracellular studies in vivo. Science 215: 1394-1396.

Aghajanian, G. K., J. M. Cedarbaum, and R. Y. Wang (1977) Evidence for norepinephrine-mediated collateral inhibition of locus coeruleus neurons. Brain Res. 136: 570-577.

Aghajanian, G. K., C. P. VanderMaelen, and R. Andrade (1983) Intracellular studies on the role of calcium in regulating activity and reactivity of locus coeruleus neurons in vivo. Brain Res. 273: 237243.

Andrade, R., and G. K. Aghajanian (1984a) Intrinsic regulations of locus coeruleus neurons: Electrophysiological evidence indicating a predominant role for autoinhibition. Brain Res. 310: 401-406.

Andrade, R., and G. K. Aghajanian (1984b) Locus coeruleus activity in vitro: Intrinsic regulation by a calcium-dependent potassium conductance but not alpha-2 adrenoreceptors. J. Neurosci. 4: 161-170.

Aston-Jones, G., and F. E. Bloom (1981) Norepincphrinc-containing locus coeruleus neurons in behaving rats exhibit pronounced responses to non-noxious environmental stimuli. J. Neurosci. 1: 877900.

Cedarbaum, J. M., and G. K. Aghajanian (1976) Noradrenergic neurons of the locus coeruleus: Inhibition by epinephrine and activation by the alpha-antagonist piperoxane. Brain Res. 112: 413-419.

Cedarbaum, J. M., and G. K. Aghajanian (1978) Activation of locus coeruleus neurons by peripheral stimuli: Modulation by a collateral inhibitory mechanism. Life Sci. 23: 1383-1392.

Engberg, G., and T. H. Svensson (1980) Pharmacological analysis of a cholinergic receptor mediated regulation of brain norepinephrine neurons. J. Neural Trans. 49: 137-150.

Ennis, M., and G. Aston-Jones (1986) Evidence for self- and neighbormediated postactivation inhibition of locus cocrulcus ncurons. Brain Res. 374: 299-305.

Foote, S. L., G. Aston-Jones, and F. E. Bloom (1980) Impulse activity of locus coeruleus neurons in awake rats and monkeys is a function of sensory stimulation and arousal. Proc. Natl. Acad. Sci. USA 77: 3033-3037.

Freedman, J. E., and G. K. Aghajanian (1984) Idazoxan (RX 781094) selectively antagonizes alpha- 2 adrenoreceptors on rat central neurons. Eur. J. Pharmacol. 105: 265-272.

Nygren, L. G., and Olson, L. (1977) Intracisternal neurotoxins and monoamine neurons innervating the spinal cord: Acute and chronic effects on cell and axon counts and nerve terminal densities. Histochemistry $52: 281-306$.

Shimizu, N., and K. Imamoto (1970) Fine structure of the locus coerulcus in the rat. Arch. Histol. Jpn. 31: 229-246.

Svensson, T. H., B. S. Bunney, and G. K. Aghajanian (1975) Inhibition of both noradrenergic and serotonergic neurons in brain by the alphaadrenergic agonist clonidine. Brain Res. 92: 291-306.

Swanson, L. W. (1976) The locus coeruleus: A cytoarchitectonic, Golgi and immunocytochemical study in the albino rat. Brain Res. 110: $39-56$.

Young, W. S. III, and M. J. Kuhar (1980) Noradrenergic alpha-1 and alpha-2 receptors: Light microscopic autoradiographic localization. Proc. Natl. Acad. Sci. USA 77: 1696-1700. 